\title{
A transition from manual to intelligent automated power system operation - a indicative review
}

\author{
Yamanappa N. Doddamani' ${ }^{1}$ U. C. Kapale ${ }^{2}$ \\ ${ }^{1}$ Government Polytechnic, India \\ ${ }^{2}$ Department of Mechanical Engineering. S. G. Balekundri Institute of Technology, India
}

\begin{tabular}{l}
\hline Article Info \\
\hline Article history: \\
Received May 2, 2018 \\
Revised Jan 16, 2019 \\
Accepted Mar 4, 2019 \\
\hline
\end{tabular}

\section{Keywords:}

Artificial intelligence Intelligent automation IOT

Power system operations

Smart grid

\begin{abstract}
This paper reviews the transition of the power system operation from the traditional manual mode of power system operations to the level where automation using Internet of Things (IOT) and intelligence using Artificial Intelligence (AI) is implemented. To make the review paper brief only indicative papers are chosen to cover multiple power system operation-based implementation. Care is taken there is lesser repeatation of similar technology or application be reviewed. The indicative review is to take only a representative literature to bypass scrutinizing multiple literatures with similar objectives and methods. A brief review of the slow transition from the traditional to the intelligent automated way of carrying out power system operations like the energy audit, load forecasting, fault detection, power quality control, smart grid technology, islanding detection, energy management etc is discussed. The Mechanical Engineering Perspective on the basis of applications would be noticed in the paper although the energy management and power delivery concepts are electrical.
\end{abstract}

Copyright $\odot 2019$ Institute of Advanced Engineering and Science. All rights reserved.

\section{Corresponding Author:}

Yamanappa N. Doddamani, Principal, Government Polytechnic, Belagavi, Karnataka, India. Email: yndabhi@rediffmail.com

\section{INTRODUCTION}

Unlike the usual review paper which intensifies in getting deeper insight on a particular domain or subdomain this review paper is indicative and thus is a broad way of glancing multiple technologies involved in power system operation which led to automation of the process. The motivation of this review paper is to generate a brief overture to the transition that occurred from the manual power system operation to the automated intelligent power system operations. The transition which highlights the transition from manual to fully automatic power system operations is given importance rather than detailing the different methods involved in the power system operations and control.

Traditional power system operations, the involvement of human intervention in many mechanical industries and manufacturing industries for energy conservation as an overall goal is dealt in this section. A duty cycle-based energy audit with rate of heat transfer, thermostat settings, outdoor temperature and audit data from the house is used to estimate the load curves of the air conditioner. The variation in thermostat for the audit is not considered for the estimation. The duty cycles are easy to model using the outdoor temperature is a random sample of households is known [1]. Hourly data of the American and Canadian control area is gathered to test the control performance of the power network. The data gathered while there is disruption caused by same network (primary) or caused by another network (secondary) for both peak and off-peak load was analyzed. The MWh components are examined for the different scenarios by designating the value system which would be carried for one-hour time. The decomposition technique used would project the MWh component into dollar worths [2]. The process of the audit included the interview with the plant office which included 10 fossils fuel-based power plants along with the site inspection, plant equipment and 
the operational data [3]. The level of reliability and the rate of heat transfer is improved [3]. A computerbased package is developed using PASCAL named Simulation for Management, Control and Analytics of Electricity End -use (SIMCA-E ${ }^{2}$ ) used or testing, performance evaluation of demand controller and control algorithms, PC based analysis for energy audit, creating different generation pattern according to different load demand requirement. Automatic logging based or manual entry of data for energy audit in SIMCA-E ${ }^{2}$ produces an effective global load curve of the facility and load profiles that have peak coincidence [4].

The electrical energy management (EEM) considering the recommended energy conservation guidelines is followed on motors, power factor and tariff control [5]. The energy audit for energy conservation in the textile industry of the state of Tamilnadu and Kerala in India with a budget constraint resulted in reduced energy cost along with ecological advantage [6]. The audit includes examination of economic usage of motors, air conditioners, lighting indoor and outdoor, electricity bill pattern, loading pattern, heating system and ventilating system [6].

\section{RECENT IMPROVEMENTS IN POWER SYSTEM OPERATIONS IN PRODUCTION INDUSTRIES}

The energy audit for reliability as the prime concern with improvement of reliability audit and annual report generation on reliability in power system is demonstrated [7]. The voltage control, security alert system, clearance time of short circuit fault, adjusting the frequency deviation are the few important criteria considered while reliability audit [7]. Energy auditing is crucial when the motor in service is included for accuracy estimation. The efficiency estimation of the in-service motor is developed by estimating the equivalent circuit parameters of the motor in service incorporating the bacterial foraging algorithm and compared with the particle swarm optimization, ant colony optimization and other traditional methods [8].

The buildings either commercial or residential comprise the key portion of the carbon footprint in the world. A system is developed named as "ECview" framework which exploits the existing power usage according to the workflow to predict the carbon emission from the building considering the weather condition in the surroundings, commuting and travel patterns and dynamic regulations from government [9].

By collecting different environmental and electrical parameters and data from different administrative units in the university a Human Computer Interface (HCI) tool is developed that tracks the ecological footprint of an institution and also indicate the scope of improvement in the footprint [10]. The centralization of the resource information in an institution along with the suggestion system is demonstrated and tested by the use of the cloud integrated overall management system. The details of each branch of the institution are centralized to be seen by the head of the institution [11]. And viewing of the summary of the observation in the internet is possible.

A smart grid environment with the advanced metering infrastructure is adopted for the intrusion detection which is a decision making of the energy theft detection from the information fusion obtained from both the sensors and the consumption data [12]. The non invasive load monitoring used in energy audit system is furthered to act as the demand response implementation both in software and hardware aspect, by exclusive scrutiny of the need of demand response [13]. The data acquisition module is connected to the event detection module which would diagnose the demand response of the system [13].

A novel hybrid classification technique is used for classifying the load identification for different combination of household appliances usage signatures. Particle Swarm Optimization based Fuzzy C means clustering with Neuro-Fuzzy classification is applied to address the ambiguities in the electrical signature sensed [14].

Power audit of a LED bulb considering the power consumption in LED wafer, phosphor coating and lamp translucent cover is experimented to find the critical area of improving the LED bulb design is identified [15]. The Home Energy Management System (EMS) for demand response in a Smart Grid environment using non intrusive load management system solving a multi-objective in-home power scheduling algorithm is established using the non-dominated sorting genetic algorithm -II without the user interference [16].

The Information technology /Information Systems investments are governed by the parameters like the behavioral economics, causality, input-output equilibrium and common conception of reduction of executive energy function. A fuzzy cognitive map is developed for the multi dimensional and non quantifiable problem of investments [17].

To satisfy the increasing demand and to provide reliable energy the synchro-phasor units are used in a wide area monitoring and control systems. Traditional manually developed rules are not suitable for bug data problems. A system for detecting the intrusion is developed involving the common path mining which is an advanced data mining technique to learn the pattern of intrusion automatically from data obtained from synchro phasor measurement unit [18]. 
The charge of electricity is dependent on the billing rate, thus to avoid the irrational concept the use and consumption of electricity is utilized. Physical condition, equipment and number of users in combination of the rate type are considered to change the actions that would lead to reduction in the cost of billing is demonstrated [19].

\section{IOT BASED POWER SYSTEM OPERATIONS IN PRODUCTION INDUSTRIES}

Low power wide area network (LPWAN) using Narrowband IOT (NB IOT) is introduced for the smart grid technology. Narrow band is chosen to avoid the traffic that is available in the crowded unlicensed band. The proposed narrow band communication is compared with the NB IOT developed by specification and facilities of LTE [20]. The IOT acts as a prospective solution in power and energy systems by real time computation capabilities, higher security, cloud connectivity and engage a seamless cooperation between the real world and internet [21]. The energy management system that would provide the dynamic control according to the generation and load, power quality problems, reliability, cost and sustainability is possible while the IOT devices are used [22].

An IPV6 communication based complete network of the IOT based smart grid conceptual model is developed considering the control devices like the switches, capacitor banks, smart sensors, reclosers and actuators as the object [23]. All the objects are connected to the central data centre from where the control signals are transmitted back as the decision making.

The IOT usage in Supervisory Control and Data Acquisition (SCADA) and Advanced Metering Infrastructure (AMI) forecast the application in future grid technology. An intelligent grid and its maintenance and development need a high-profile leadership with good technical workforce on the IOT in order to execute and maintain the implementation [24].

Internet Protocol Smart Objects (IPSO), European Telecommunications Standards Institute (ETSI), Zigbee are working on the feasibility of the potential usage of these technologies on the IOT applications [25]. The dependent sensors that changes its values with change in the other sensors value is identified by collecting data from the network of sensors, and updated in the cloud space, the data which could be used to guess the sensor value in case of sensor failure [26]. The speed of updation of the physical data on to the internet is discussed followed by the survey of future of IOT and its challenges [27]. A cuttingedge technology called Fog computing, which is an implementation that uses the end user clients or near user edge devices to use those nodes as the storage unlike using the cloud space, is discussed [28]. Fog computing guarantees real time decision making using data analytics in IOT's set up in larger geographical areas. A theoretical model of Fog computing implementation is develop facilitating the comparison between the cloud computing platform and inferred that the energy cost of using Fog computing would reduce $40.48 \%$ of the cost unlike while using cloud computing [29].

\section{FROM ARTIFICIAL INTELLIGENCE TO AUTOMATED INTELLIGENCE}

A different security problem that may occur along with different security technologies that can be implemented is discussed [30]. The data analytics for the data collected either in the cloud or the fog computing would need the Artificial Intelligence for inference or decision making. The intelligent control of wind energy conversion systems with fault pattern identification of the Smart Grid system using Artificial Intelligence on a real time simulator is presented [30].

A machine intelligence-based ship navigation strategy selection is developed to have better energy efficiency which includes the data expansion, integrity verification and data regression steps [31]. Applications of intelligence in the smart grid with renewable energy sources using sophisticated communication and data processing techniques to have a controlled power quality and reliability which involves islanding, storage management and important automation in the power system is reviewed [32]. The stress on adaptation of the renewable based sources and the usage of direct DC grid is taken into account for the intelligent DC homes considering both intelligence and energy efficiency [33]. The vulnerabilities occurring while applying the intelligence and automation on smart grid applications caused by the public communication infrastructure and the internet-based protocols is discussed in an IOT environment [34].

A solution that avoids the bad data detection by estimating any one of the DC or AC state estimation is proposed [34]. Load forecasting is applied including the complex external factors like the climate and social convention using the deep learning technologies in IOT for accurate future load estimation [35]. Sensing systems advancement to control the energy flow direction in the grid efficiently, abiding the IEEE standard 1459-2010 for analyzing the voltage and current signals, by means of applying the suitable decision criteria using the IOT implementation [36]. A co simulator is developed to evaluate different IOT aided control algorithms, for scheduling the energy consumption which can be implemented by utility companies 
and strategy initiator [37]. By applying the IOT technology the context aware technology is developed which would use the smart meters to find out the flow energy from the grid and to the grid from the renewable energy produced from the homes installed with environment friendly power generators. A day ahead pricing scheme is developed by the use of the dynamic pricing scheme in an optimization dual problem [38].

An interface between the AI applications and the EMS for intelligent alarm processing, fault diagnosis and power system restoration are presented for a power system model and three different EMS architecture is developed for a common system [39]. There is a cognitive barrier that is faced by most of the power system operators due the large inrush of data from the different portion of the power system while there is an emergency. A relay fault diagnosis system is illustrated using the AI which solves the decision making barrier which occurred during the usage of traditional numerical method [40].

The literature review of different applications of AI tools used in the power industry in Japan, proving that Japan were more widespread in applying Artificial Intelligence by citing papers that were developed by the industry people and collaborative research involving industry [41]. A cooperating architecture called cooperative intelligent real time control architecture (CIRCA) which compensates the reactivity issue of the AI by creating real time subsystems for a better performance goals including precision, completeness of output and timeliness [42].

An Artificial Neural Network (ANN) based load forecasting considering the relation between the load and the temperature given the season, day type, hour of the day is implemented that would forecast the load before 24 hours [43]. The dynamic load modeling of the power system for carrying out stability analysis is implemented by using the ANN [44]. A computer program that would find the problems due to harmonics by getting the inputs like the topology, equipment, type and ratings, power signature, symptoms, operating practices etc., using the expert system implementation using AI [45]. An optimal online reactive power control technique is implemented by considering an uncertain reactive load applied. ANN enhanced by fuzzy sets is used to determine the membership of the control variables for different load changes [46].

The voltage collapse is predicted using AI due to the vulnerability on power system, which causes local voltage instability and security issues. The indices of voltage security and the local voltage collapse is predicted with high robustness, by giving the real power, reactive power and voltage (PQV) surface as the input to the AI [47]. The manual supervision of the power system is automated by applying the AI technique which not only considers the local fault clearance but also supervises the voltage levels, harmonics and power factor of the desired state while making the decision unlike the manual supervision [48].

The AI model for non parametric approach called as adaptive Back Propagation network is incorporated for applying the load modeling for transient stability analysis, which gave accurate resemblance with the actual field test data in a system in china [49]. Replacing the measurement-based load modeling which only concentrates on the symmetrical disturbance in three phases an asymmetrical load modeling is developed without the use of extra data acquisition equipments. A tool called COMTRADE is used to acquire data and infer the asymmetrical load modeling [50].

A DSTATCOM control based on artificial immune system (AIS) for robust control in order to maintain the constant voltage at the PCC for eliminating the power quality issue due to pulse loads in the on ship electric system [51]. A DSP based implementation of the ANN controlled Unified Power Quality Conditioner (UPQC) is developed which is trained from the logged data received from the PI controller implementation [52] for performance improvement.

Fuzzy inference system or Genetic Algorithm is used to develop the gain parameter estimation in the automatic generation control (AGC) in the load frequency control (LFC) making a balance between generation and demand [53]. AIS based controller for the generator excitation system to compensate the high energy loads. The parameter for the controller is optimized for the best control action on the generator excitation controller during the disturbances [54]. Genetic Algorithm based optimal layout of the offshore wind farm along with the line connection topology optimized using ant colony optimization algorithm. The technique proposes an efficient and economical wind farm [55]. The dynamic characteristics of the load which is extremely non linear and time varying and thus must be modeled accurately. An Improved Particle Swarm Optimization (IPSO) parameter estimation method is used to accurately determine the load model using AI based load modeling [56]. Actuator and sensor fault detection with low computational cost is developed using a novel AI method which uses single fault detection estimator instead of bank of estimators employed in traditional methods [57]. The literature talks about the different intelligent techniques that can be used to gather deeper meaning of the big data obtained from different source like physical social and cyber environment [58]. A random matrix theory-based implementation for big data analytics on a smart grid power system is carried out with situation awareness sensitivity and feasibility to be implemented on real time smart grid systems [59]. Artificial immune system (AIS) based load forecasting is implemented and compared with the neural network, autoregressive integrated moving average, and exponential smoothing methods and found to be dominating in performance [60]. 


\section{CONCLUSION}

A brief review of literature involving the traditional way of power system operation in manufacturing and mechanical industries, and a slow improvement to the advanced power system operation methods are introduced. The power system operations like the energy audit, load forecasting, energy management, load modeling, smart grid, load frequency control, power quality improvement, microgrid applications are the few that are analyzed to realize the transition from the traditional way of power system operation to the power system operation carried out using advanced intelligent based, IOT based and Big data-based operations. This is an indicative review which gives the reader a view of how well the power system operation in industries can be automated intelligently in future.

\section{REFERENCES}

[1] J. H. Reed, R. P. Broadwater, A. Chandrasekaran, and A. Oka, "Predicting Air Conditioner Load Curves from Energy Audit Data: A Comparison of Predicted and Actual Air Conditioning Data from the Athens Load Control Experiment." IEEE Transactions on Power Systems, vol. 5, no. 2, pp 359-365, May 1990.

[2] Cohn, "Auditing Control Performance of Interconnected Areas Utilizing the Components Concept and Its Decomposition and Evaluation Techniques. Part I: Audit Technology and Field Tests. Part II: Test Results," IEEE Transactions on Power Systems, vol. 3, no. 2, pp. 581-603, May 1988.

[3] J.R. Wilkinson, "Comprehensive performance audit of a utility", IEEE Transactions on Energy Conversion, vol. 6, no. 2, pp 243-250, Jun 1991.

[4] A. Martins, H. Jorge, J. Mota, R. ParTacho, A. Gomes, "A PC-Based Simulation Package for Supporting End-User Demand Side Management Strategies," IEEE Trans. on Power Systems, vol. 6, no. 3, pp. 897-903, Aug 1991.

[5] A. Z. Khan, "Electrical energy conservation and its application to a sheet glass industry," IEEE Trans. Energy Conversion, pp. 666-671, Sep. 1996.

[6] C. Palanichamy, C. Nadarajan, P. Naveen, N. S. Babu and Dhanalakshmi, "Budget constrained energy conservation - an experience with textile industry," IEEE Trans. On Energy Conversion, vol. 16, no. 4, Dec 2001.

[7] J. M. Tesseron, "Mission: Reliability," IEEE Power and Energy Magazine, vol. 6, no. 1, pp. 42-48, Jan - Feb 2008.

[8] V. P. Sakthivel, R. Bhuvaneswari, and S. Subramanian, "Non-intrusive efficiency estimation method for energy auditing and management of inservice induction motor using bacterial foraging algorithm," IEEE ProceedingsElectric Power Applications, vol. 4, no. 8, pp. 579-590, 2010.

[9] G. Thiagarajan, V. Sarangan, R. Suriyanarayanan, P. Sethuraman, A. Sivasubramaniam, A. Yegyanarayanan, "Automating a buildings carbon management," IEEE Computer, vol. 44, no. 1, pp. 24-30, Jan 2011.

[10] D. B. Heras, D. Otero, and F. Arguello, "An eco-feedback system for improving the sustainability performanceof universities," in Proc. 2011 IEEE International Conference on Virtual Environments Human-Computer Interfaces and Measurement Systems, Ottawa, ON, 2011, pp. 1-6.

[11] Mary Jane Magno-Tan, Allan V. Crisostomo, Bill Villaflor, James C. Faller, "Cloud-Based College Management Information System for Universities," International Journal of Information and Education Technology, vol. 4, pp. 508, 2014.

[12] S. McLaughlin, B. Holbert, A. Fawaz, R. Berthier, S. Zonouz, "A multi-sensor energy theft detection framework for advanced metering infrastructures," IEEE J. Sel. Areas Commun., vol. 31, no. 7, pp. 1319-1330, Jul 2013.

[13] D. He, W. Lin, N. Liu, R. G. Harley, T. G. Habetler, "Incorporating non-intrusive load monitoring into building level demand response," IEEE Trans. Smart Grid, vol. 4, no. 4, pp. 1870-1877, Dec 2013.

[14] Y. H. Lin, M. S. Tsai, "Non-intrusive load monitoring by novel neuro-fuzzy classification considering uncertainties," IEEE Trans. Smart Grid, vol. 5, no. 5, pp. 2376-2384, Sep 2014.

[15] Sinan Li, "Power Flow Analysis and Critical Design Issues of Retrofit Light-Emitting Diode (LED) Light Bulb," IEEE Transactions on Power Electronics, vol: 30, no. 7, pp. 3830-3840, Jul 2015.

[16] Y. H. Lin, M. S. Tsai, "An advanced home energy management system facilitated by nonintrusive load monitoring with automated multiobjective power scheduling," IEEE Trans. Smart Grid, vol. 6, no. 4, pp. 1839-1851, Jul 2015.

[17] Z. Irani, A. M. Sharif, T. Papadopoulos, "Organizational energy: a behavioural analysis of human and organizational factors in manufacturing," IEEE Trans. Eng. Manag., vol. 62, no.2, pp. 193-204, 2015.

[18] S. Pan, T. Morris, U. Adhikari, "Developing a hybrid intrusion detection system using data mining for power systems," IEEE Trans. on Smart Grid, vol. 6, no. 6, pp. 3104-3113, 2015.

[19] Jesus Martinez Patino, et al, "A modified analysis of electrical energy consumption in University buildings," IEEE Latin America Transactions, vol. 15, no. 3, pp. 408 - 414, Mar 2017.

[20] Yuke Li, "Smart Choice for the Smart Grid: Narrowband Internet of Things (NB-IoT)," IEEE Internet of Things Journal, vol. 5, no. 3, pp. 1505 - 1515, 2018.

[21] G. Bedi, G. K. Venayagamoorthy and R. Singh, "Navigating the challenges of Internet of Things (IoT) for power and energy systems," 2016 Clemson University Power Systems Conference (PSC), Clemson, SC, 2016, pp. 1-5.

[22] G. Bedi, G. K. Venayagamoorthy and R. Singh, "Towards an Intelligent Power Network with Internet of Things (IoT)," accepted for publication in Intelligent Systems Conference 2017. 
[23] Al-Ali A.R. and Aburukba R., "Role of Internet of Things in the Smart Grid Technology," Journal of Computer and Communications, vol. 3, pp. 229- 233, 2015.

[24] R. Young, J. McCue and C. Grant, "The power is on: How IoT technology is driving energy innovation," Deloitte Center for Energy Solutions Report, Jan 2016, http://dupress.com/articles/internet-ofthings-iot-in-electric-powerindustry/

[25] N. Bui, A. P. Castellani, P. Casari and M. Zorzi, "The internet of energy: a web-enabled smart grid system," in IEEE Network, vol. 26, no. 4, pp. 39-45, 2012.

[26] P. K. Choubey, S. Pateria, A. Saxena, Vaisakh Punnekkattu Chirayil SB, K. K. Jha and Sharana Basaiah PM, "Power efficient, bandwidth optimized and fault tolerant sensor management for IOT in Smart Home," 2015 IEEE International Advance Computing Conference (IACC), Banglore, 2015, pp. 366-370.

[27] V. Gazis, "A Survey of Standards for Machine to Machine (M2M) and the Internet of Things (IoT)," in IEEE Communications Surveys \&Tutorials, vol. 19, no. 1, pp.1-1, Jul 2016.

[28] M. Yannuzzi, R. Milito, R. Serral-Gracià, D. Montero and M. Nemirovsky, "Key ingredients in an IoT recipe: Fog Computing, Cloudcomputing, and more Fog Computing," 2014 IEEE 19th InternationalWorkshop on Computer Aided Modeling and Design of Communication Links and Networks (CAMAD), Athens, 2014, pp. 325-329.

[29] S. Sarkar and S. Misra, "Theoretical modelling of fog computing: a green computing paradigm to support IoT applications," in IET Networks, vol. 5, no. 2, pp. 23-29, 2016.

[30] K. Lee, D. Kim, D. Ha, U. Rajput and H. Oh, "On security and privacy issues of fog computing supported Internet of Things environment," 2015 6th International Conference on the Network of the Future (NOF), Montreal, QC, 2015, pp. 1-3.

[31] Lokukaluge Prasad Perera and Brage Mo, "Machine Intelligence Based Data Handling Framework for Ship Energy Efficiency," IEEE Transactions on Vehicular Technology, vol. 66, no. 10, pp. 8659 - 8666, Oct 2017.

[32] T. Strasser, "A review of architectures and concepts for intelligence in future electric energy systems," IEEE Trans. Ind. Electron., vol. 62, no. 4, pp. 2424-2438, Apr 2015.

[33] E. Rodriguez-Diaz, J.C. Vasquez, J.M. Guerrero, "Intelligent DC Homes in Future Sustainable Energy Systems: When efficiency and intelligence work together," Consumer Electronics Magazine IEEE 5.1, pp. 74-80, Jan 2016.

[34] K. Wang, H. Li, Y. Feng, G. Tian, "Big data analytics for system stability evaluation strategy in the energy internet," IEEE Trans. Ind. Informat., vol. 13, no. 4, pp. 1969-1978, Aug 2017.

[35] L. Li, K. Ota, M. Dong, "When Weather Matters: IoT-Based Electrical Load Forecasting for Smart Grid," IEEE Commun. Mag., vol. 55, no. 10, pp. 46-51, Oct 2017.

[36] Rosario Morello, et al, "A Smart Power Meter to Monitor Energy Flow in Smart Grids: The Role of Advanced Sensing and IoT in the Electric Grid of the Future," IEEE Sensors Journal, vol. 17, no. 23, pp. 7828-7837, Dec 2017.

[37] Xin Li, Qiuyuan Huang, Dapeng Wu, "Distributed Large-Scale Co-Simulation for IoT-Aided Smart Grid Control," IEEE Access, vol. 5, pp. 19951-19960.

[38] T. C. Chiu, Y. Y. Shih, A. C. Pang, C. W. Pai, "Optimized day-ahead pricing with renewable energy demand-side management for smart grids," IEEE Internet of Things Journal, vol. 4, no. 2, pp. 374-383, Apr 2017.

[39] J. J. Bann, "Integrating AI applications in an energy management system," IEEE Expert, vol. 12, no. 6, pp. 53-59, 1997.

[40] B. F. Wollenberg, T. Sakaguchi, "Artificial Intelligence in Power System Operations," Proceedings of the IEEE, vol. 75, no. 12, pp. 1678-1685, Dec 1987.

[41] S. Rahman, "Artificial intelligence in electric power systems: a survey of the Japanese industry," IEEE Trans on Power Syst., pp. 1211-1218, 1993.

[42] D. Musliner, E. Durfee, K. Shin, "Circa: A cooperative intelligent real-time control architecture," IEEE Transactions on Systems Man and Cybernetics, vol. 23, no. 6, 1993.

[43] M. Djukanovic, B. Babic, D. J. Sobajic, Y. H. Pao, "Unsupervised/supervised learning concept for 24-hour load forecasting," IEEE Proceedings-C, vol. 140, no. 4, pp. 311-318, Jul 1993.

[44] B.-Y. Ku, R.J. Thomas, C.-Y. Chiou, C.-J. Lin, "Power system dynamic load modeling using artificial neural networks," IEEE Transactions on Power Systems, vol.9, no.4, pp.1868-1874, Nov 1994.

[45] D. D. Shipp, W. Vilchek, M. E. Swartz, N. H Woodly, "Expert System for Analysis of Electric Power System Harmonics," IEEE Industry Applications Magazine, 1995.

[46] K. H. Abdul-Rahman, S. M. Shahidepour, M. Daneshdoost, "AI Approach to Optimal Var Control with Fuzzy Reactive Loads," IEEE PES Winter Meeting, 1994.

[47] K. Yabe, J. Koda, K. Yoshida, K. H. Chiang, P. S. Khedkar, D. J. Leonard, N. W. Miller, "Conceptual designs of AI-based Systems for local prediction of voltage collapse," IEEE Trans. Power Syst., vol. 11, no. 1, pp. 137-145, Feb 1996.

[48] M.S. Ayala, G.J. Botura and O.A. Maldonado "An AI tool for supervising substations," IEEE Potentials, vol. 20, no. 5, pp. 13-18, Dec 2001/Jan 2002.

[49] A. Qian, D. Gu, C. Chen, "New load modeling approaches based on field tests for fast transient stability calculations," IEEE Transactions on Power Systems, vol. 21, pp. 1864-1873, Nov 2006.

[50] W. Xu, C. Chen, Q. Ai, W. Wang, X. Ling, B. Liu, C. Wang, "Load modeling during asymmetric disturbance in a metropolitan power grid," IEEE Trans. Power Syst., vol. 24, no. 3, pp. 1191-1873, Aug. 2009.

[51] P. Mitra, G. K. Venayagamoorthy, "An adaptive control strategy for DSTATCOM applications in an electric ship power system," IEEE Trans Power Electronics, vol. 25, no. 1, pp. 95-104, Jan 2010. 
[52] Vadirajacharya G. Kinhal, Promod Agarwal, Hari Oam Gupta, "Performance Investigation of Neural-NetworkBased Unified Power-Quality Conditioner," IEEE Transactions on Power Delivery, vol. 26, no. 1, pp. 431-437, Jan 2011

[53] L. Chang-Chien, Y. Wu, J. Cheng, "Online estimation of system parameters for artificial intelligence applications to load frequency control," IET generation transmission \& distribution, vol. 5, no. 8, pp. 895-902, 2011.

[54] C. Yan, G. K. Venayagamoorthy, "AIS-Based coordinated and adaptive control of generator excitation systems for an electric ship," IEEE Trans. Ind. Electron., vol. 59, no. 8, pp. 3102-3112, Aug 2012.

[55] Y. K. Wu, et al., "Optimization of the wind turbine layout and transmission system planning for a large-scale offshore wind farm by AI technology," IEEE Trans. Ind. Appl., vol. 50, no. 3, pp. 2071-2080, 2014.

[56] P. Regulski, D. S. Vilchis-Rodriguez, S. Djurovic, V. Terzija, "Estimation of composite load model parameters using an improved particle swarm optimization method," IEEE Trans. Power Del., vol. 30, no. 2, pp. 553-560, Apr 2015.

[57] Konstantinos Michail, et al, "AI-Based Actuator/Sensor Fault Detection with Low Computational Cost for Industrial Applications," IEEE Transactions on Control Systems Technology, vol. 24, no. 1, pp. 293-301, Jan 2016.

[58] S. Amit, "Internet of Things to smart IoT through semantic cognitive and perceptual computing," IEEE Intell. Syst., vol. 31, pp. 108-112, 2016.

[59] X. He, Q. Ai, R. C. Qiu, W. Huang, L. Piao, H. Liu, "A big data architecture design for smart grids based on random matrix theory," IEEE Trans. Smart Grid, pp.674-686, Jan 2017.

[60] Dudek G., "Artificial immune system with local feature selection for short-term load forecasting," IEEE Trans. Evol. Comput., vol. 21, no. 1, pp. 116-130, 2017.

\section{BIOGRAPHIES OF AUTHORS}

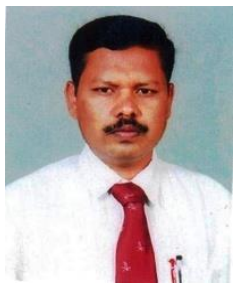

Yamanappa N. Doddamani currently working as the Principal in Governtment Polytechnic, Belagavi, has 26 years of teaching experience at different levels completed his M. Tech in National Institute of Technology in the year 2004 and currently pursuing his PhD in Electrical Engineering in VTU Belagavi. His research interests are energy audit and mechanical engineering, Internet of Things (IOT).

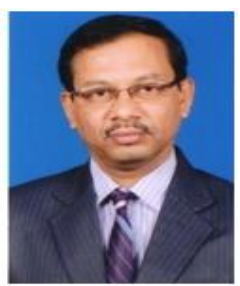

Dr. U. C. Kapale, has pursued B.E. (Mech.; 1988), M.E. (Thermal Power Engg.; 1994) from PDA College of Engg., Kalburgi, and Ph. D. (Heat Power; 2007) from MNNIT, Allahabad. He has 30 years of teaching and 01-year industrial experience. He has contributed 12 research papers in International journal and 04 research papers in National journal. At present 08 candidates are pursuing $\mathrm{Ph}$. D. under his guidance and presently working as Professor, Head, Mech. Engg. and Dean (Acad.) at SSET'S. S. G. Balekundri Institute of Technology, Belagavi, Karnataka. 